\title{
Mediator Role of Psychological Flexibility in the Relationship Between Traumatic Grief and Psychological Symptoms
}

\author{
Samet BAŞ*, Selva ÜLBE, Özge YÜKSEL, Duygu GÜNGÖR \\ *Dokuz Eylul University, Department of Psychology, Izmir, Turkey / sametbas@yahoo.com
}

\section{INTRODUCTION}

When a loved person is lost, grief related feelings such as sadness, anxiety, pain and emptiness are inevitably experienced. In general, bereaved people can successfully deal with grieving process over time.

However, especially when the loss is traumatic, it can be difficult to adapt to changing life and maintain mental health. In this regard, some of the recent research indicated that the acceptance of such a traumatic event or showing psychological flexibility which is a main factor of Acceptance and Commitment Therapy (ACT) could be considered as a protective factor against psychological symptoms.

\section{AIM OF THE STUDY}

The present study aims at testing whether psychological flexibility is a mediator of the relationship between traumatic grief and negative psychological symptoms in a Turkish sample.

\section{METHOD}

384 bereaved individuals $(76 \%$ females) completed Turkish version of following questionnaires. In the current study a data which is some part of a comprehensive grief project was used.

Traumatic Grief Inventory-Self Report (TGI-SR; Boelen et al., 2018)

Acceptance and Action Questionnaire-II (AAQ-II; Bond et al., 2011)

Depression Anxiety Stress Scale (DASS- 21; Henry \& Crawford, 2005)

\begin{tabular}{|l|c|c|c|c|}
\hline Table 1: Descriptive Statistics \\
\hline & Min & Max & M & Sd \\
\hline Age & 18.0 & 74.0 & 30.4 & 11.8 \\
\hline $\begin{array}{l}\text { Time Since Loss } \\
\text { (Month) }\end{array}$ & 3.1 & 71.0 & 28.3 & 18.7 \\
\hline
\end{tabular}

\section{RESULTS \& CONCLUSION}

Three separate mediation analyses which were computed using PROCESS for SPSS (Hayes, 2013) indicated that psychological flexibility (partially) mediated all relationships between traumatic grief and depression, anxiety and stress (Table 2).

Such findings emphasize the critical role of psychological flexibility as a protective factor against to psychological symptoms for bereaved individuals at risk of traumatic grief. In this regard, psychotherapeutic tools of ACT based interventions appear as a new alternative treatment option for bereaved individuals.

Table 2: Summary of Total, Indirect, and Direct Effects of Mediation Models

\begin{tabular}{|c|c|c|c|c|c|c|}
\hline Outcome Variable & \multicolumn{2}{|c|}{ Depression } & \multicolumn{2}{|c|}{ Anxiety } & \multicolumn{2}{|r|}{ Stress } \\
\hline $\begin{array}{l}\text { Predictor } \\
\text { Variable: TGI-SR }\end{array}$ & b & $95 \% \mathrm{Cl}$ & b & $95 \% \mathrm{Cl}$ & b & $95 \% \mathrm{Cl}$ \\
\hline Total & .19 & {$[.16, .22]$} & .16 & {$[.13, .19]$} & .16 & {$[.14, .19]$} \\
\hline Indirect (AAQ-II) & .09 & {$[.07, .12]$} & .07 & {$[.06, .10]$} & .08 & {$[.06, .10]$} \\
\hline Direct & .10 & {$[08, .13]$} & .09 & {$[.06, .11]$} & .08 & {$[.05, .11]$} \\
\hline$R^{2}$ & & .32 & & .27 & & .26 \\
\hline Sobel Test (z) & & 8.51 & & 7.84 & & 8.23 \\
\hline
\end{tabular}

Note: Bold values show significant effects determined by a $95 \%$ bias corrected bootstrapped confidence intervals (1000 bootstrapped samples)

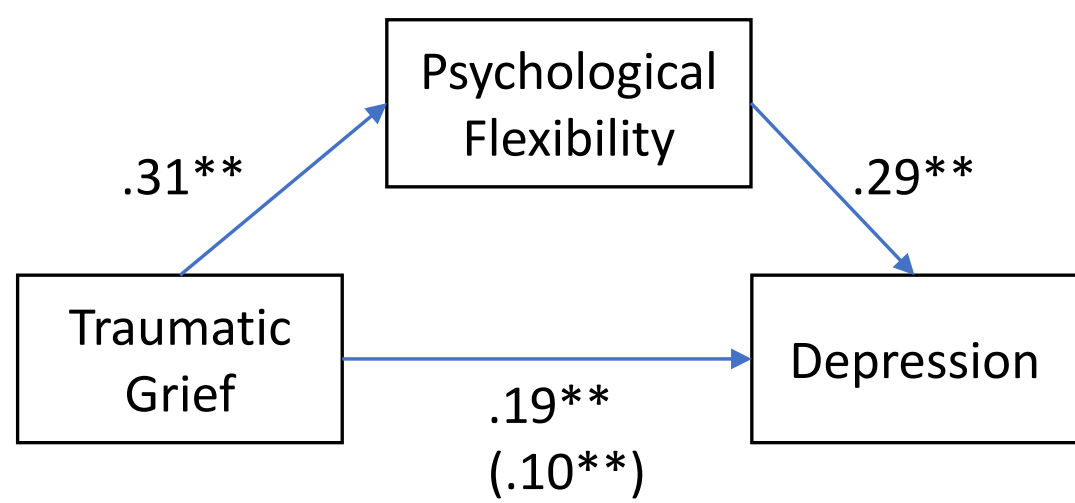

Figure 1: Mediation analysis model between psychological flexibility and depression $* * p<0.001$

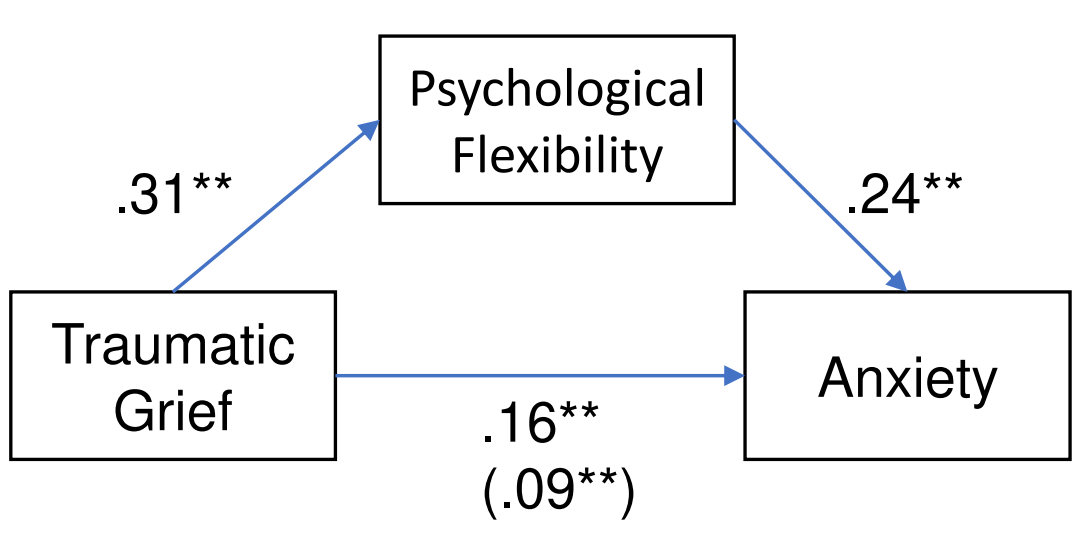

Figure 2: Mediation analysis model between psychological flexibility and anxiety $* * p<0.001$

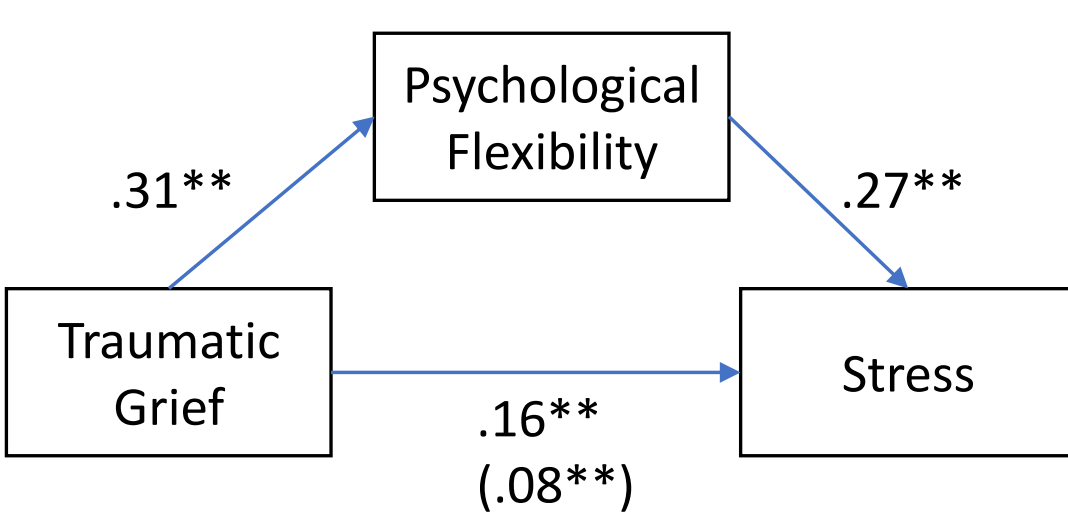

Figure 3: Mediation analysis model between psychological flexibility and stress $* * p<0.001$ 